Gino Guillermo Tapia-Zegarra 1

Jesús L. Chi rinos 1,2

Lenibet Miriam Tapia-Zegarra 3

\section{Factores contribuyentes al desarrollo de infecciones adquiridas en la comunidad en diabéticos tipo 2 admitidos en salas de medicina del Hospital Nacional Cayetano Heredia}

\author{
Factors contributing to the development \\ of community-acquired infections in type-2 \\ diabetics admitted to the Cayetano Heredia \\ National Hospital
}

1 Departamento Académico de Salud Pública, Universidad Peruana Cayetano Heredia. Av. Honorio Del gado 430 San Martin de Porras, Lima 31, Perú. ginotapia@hotmail.com 2 Departamento Académico de Medicina, Universidad Peruana Cayetano Heredia Av. Honorio Del gado 430, San Martin de Porras, Lima 31, Perú.

3 Universidad Nacional Federico Villareal. 3230 So. Gessner Rd, Apt \#2716, Houston TX 77063, U. S. A.
Abstract This study aims to identify factors contributing to the development of community-acquired infections in hospitalized type- 2 diabetics, using an unmatched case-control design. A total of 105 pairs of diabetic patients with community-acquired infections as compared to non-infectious di seases, respectively, were chosen randomly from the discharge registry of the clinical wards of a teaching hospital from 1991 to 1998. Bivariate and multivariate analysis was conducted with estimation of the adjusted odds ratio. According to stratified analysis controlling for age and sex, autonomi c neuropathy proved to be a contributing factor to development of urinary tract infections $(O R=4.07)$. In the multivariate model, peripheral vasculopathy was i sol ated ( $R 2$ : 0.24 ) as a contributing factor in the development of soft-tissue infecti ons (OR $=6.79$ ). Confidence intervals were si gnificant at 0.05 . In concl usi on, peri pheral vasculopathy and neurogenic bladder contribute to the development of community-acquired infections in hospitalized type-2 diabetics.

Key words Non-Insulin-Dependent Diabetes Mellitus; Infection; Epidemiology

Resumen El propósito del estudio es identificar factores contribuyentes al desarrollo de infecciones adquiri das en la comuni dad en diabéticos ti po 2 hospitalizados. De tal forma, se siguió el diseño de un estudio de casos y controles no apareado. Consecuentemente, se eligieron en forma aleatoria 105 diabéticos con infección adquirida en la comunidad y 105 diabéticos con patología no infecciosa, obtenidos de los Servicios de Medicina de un hospital universitario, entre 1991 y 1998. Mediante el análisis bivariado y multivariado, se obtuvo la estimación ajustada del vaIor de odds ratio. Los resultados permiti eron, según análi sis estratificado por edad y sexo, obtener a neuropatía autonómica como factor contribuyente de infecciones urinarias $(\mathrm{OR}=4,07)$. En el modelo multivariado se aisló a vasculopatía periférica $(R 2: 0,24)$ como contribuyente de infección de piel y partes blandas $(\mathrm{OR}=6,79)$. Los interval os de confianza fueron significativos y de 95\% de confiabilidad. En conclusión, vasculopatía periférica y vejiga neurogénica contribuyen al desarrollo de infecci ones adquiridas en la comunidad en diabéticos tipo 2 hospital izados. Palabras clave Diabetes Mellitus No Insulino-Dependiente; Infección; Epidemiología 


\section{Introducción}

La diabetes mellitus es considerada actualmente un problema de Salud Pública. La prevalencia de diabetes mellitus (tipo 1 y 2 ), de casos nuevos varía entre 2 y $5 \%$ de la población mayor de 30 años de edad a nivel mundial, constituyendo la diabetes tipo 2 entre el 85$95 \%$ de los casos (Pfizer, 1982). En un estudio multicéntrico en nueve ciudades capitales del Brazil, en individuos entre los 30-69 años de edad, se halló una prevalencia ajustada de diabetes mellitus en general de 7,6\% entre casos nuevos y pre-diagnosticados (Malerbi \& Franco, 1992). En el Perú, la prevalencia ajustada de diabetes mellitus en general es de 1 a $8 \%$ de la población mayor de 18 años de edad, incluyendo casos auto notificados y nuevos, siendo Lima una de las ciudades más afectadas $(7,6 \%)$ (Seclén et al., 1999). La importancia de esta enfermedad radica en sus complicaciones crónicas microvasculares, como daño neuropático, y macrovasculares a nivel periférico, cerebral y coronario (Fore, 1995).

Las infecciones son complicaciones agudas muy frecuentes y severas en diabéticos ambulatorios y hospitalizados (File Jr. \& Tan, 1997). Los principales factores que predisponen a los diabéticos a las infecciones son el daño a la barrera primaria que forma la piel por isquemia o trauma, frecuentes secundarias a neuropatía, alteración de la función inmunológica humoral y celular, colonización de piel por gérmenes más patógenos, enfermedad micro y macrovascular, mal nutrición, hiperglicemia, instrumentación, catéteres, saneamiento ambiental deficiente y grado de inmadurez individual (Eliopoulos, 1995). La complicación más importante en población diabética hospitalizada de países subdesarrollados, así como la de algunos países desarrollados, es la infección (Contreras, 1993; De Aguilar et al., 1997; Villena et al., 1996). En ciudades del Perú, esta prevalencia de infecciones en diabéticos hospitalizados figura entre el $29,8 \%$ y el $59,2 \%$ de diabéticos tipo 2, según datos de hospitales en la Capital y en la provincia del Perú, respectivamente (Concha, 1995; Villena et al., 1996). Sin embargo, en países desarrollados, la mayor causa de mortalidad se debe a complicaciones macrovasculares, en particular a enfermedad coronaria (Balkau et al., 1993). Se ha publicado escasamente en forma específica sobre el grado de asociación de infecciones en relación a factores demográficos, clínicos y de laboratorio (Concha, 1995; Marulanda \& De Martinez, 1989). Al gunos de estos factores contribuyentes son los que deseamos identificar en la po- blación de un hospital de referencia de Lima, con el fin de establecer la base sobre la cual ubicar grupos con mayor predisposición al desarrollo de infecciones, para instituir medidas de prevención y tratamiento oportunos en ellos.

El objetivo del presente estudio es determinar los factores sociodemográficos (analfabetismo, hacinamiento no crítico), clínicos (desnutrición, vasculopatía periférica, neuropatía periférica, vejiga neurogénica) y de laboratorio (inversión de la relación albúmino/globulina séricas, creatinina sérica elevada) contribuyentes al desarrollo de infecciones adquiridas en la comunidad en diabéticos tipo 2 que se hospitalizan en un hospital de cuarto nivel de la Capital del Perú.

\section{Materiales y métodos}

Tipo de estudio

Se realizó un estudio de Casos y Controles, no apareado con una razón de selección 1 a 1 .

Selección de la población

- Tamaño muestral

Para un error alfa del $5 \%$, error beta del $10 \%$, detección de una odds ratio (OR) de 2,5 y una frecuencia del $30 \%$ de la enfermedad en estudio dentro de la población a investigar (Villena et al., 1996), se deberán escoger 105 casos y 105 controles (Schlesselman, 1982).

\section{- Marco muestral e instrumento metodológico}

a) Criterios de inclusión: pacientes no gestantes de 30 ó más años de edad vivos al alta, que se hospitalizaron en los Servicios de Medicina General, Tropical y Unidad General de Cuidados Intensivos ( $\mathrm{UCl}$ ) del Hospital Nacional Cayetano Heredia $(\mathrm{HNCH})$ durante el período enero 1991-diciembre 1998, con diagnóstico de diabetes mellitus tipo 2 (antecedente de diagnóstico por un médico, síntomas de poliuria, polidipsia y perdida ponderal, glicemia de ayunas y/o hemoglobina glicosilada en algunos casos) confirmado durante la hospitalización, con y sin presencia concomitante del diagnóstico clínico al alta de infección adquirida en la comunidad, como motivo o no de hospitalización, y aquellos con evaluación ambulatoria durante los siguientes 2 a 3 meses después del alta. 
b) Criterios de exclusión en ambos grupos: todo aquel paciente menor de 30 años de edad, sin diagnóstico definido de diabetes mellitus tipo 2, gestante, pacientes con hiperglicemia no diabética (por otras enfermedades u drogas, a excepción de las tiazidas y furosemida, por formar frecuentemente parte del armamentario terapeútico de los pacientes en el estudio), enfermedades causantes de inmunosupresión secundaria o causantes de alteración en las barreras normales a la infección u obstrucción de vías anatómicas y candidiasis mucocutánea, por no ser la hiperglicemia una variable de estudio, y escasos datos por falta de una sistemática evaluación diagnóstica en los pacientes internados en el $\mathrm{HNCH}$ como el caso de esofagitis y vulvovaginitis, y los diagnósticos de parasitosis intestinal por falta de evaluación sistemática de igual forma.

c) Metodología: se revisaron las historias clínicas de aquellos pacientes seleccionados, considerándose únicamente los datos de la primera hospitalización. Se incluyeron las historias según elección al azar del total disponible para el período del estudio, hasta completar por saturación el número calculado de 105 pacientes para cada grupo.

d) Evaluaciones ambulatorias: empleadas para suplir la falta de evaluación de alguna variable de estudio dentro de la hospitalización o cuando su interpretación fuese más significativa fuera del proceso de descompensación de la enfermedad de fondo, como es el caso de creatinina sérica y hemoglobina glicosilada. Se emplearon hojas de control metabólico ambulatorio de consulta externa 2 a 3 meses antes o después de la hospitalización, según sean datos positivos de diagnóstico previos a la hospitalización o negativos de diagnóstico posterior a la hospitalización, respectivamente.

\section{- Tipología de variables}

La variable dependiente fue diabetes mellitus tipo 2 con el concomitante diagnóstico clínico de infección adquirida en la comunidad. Se definió infección adquirida en la comunidad como aquella infección con evidencia de estar presente o incubándose al momento de la admisión (primeras 48 horas) o aquella infección que se presenta fuera de las primeras semanas (consideraremos al menos cuatro semanas) luego de alguna hospitalización previa (Garner et al., 1988). Se incluyeron para el estudio aquellas infecciones que sean elegibles para la exposición (Lasky \& Stolley, 1996), es decir que ocurran en mayor frecuencia en diabéticos o aquellas cuya patogénesis hacia la producción de enfermedad se vea facilitada por las alteraciones orgánicas estructurales y funcionales propias de la enfermedad de fondo, incluyéndose de esta manera la infección de partes blandas, ótica, ocular, meningoencefálica, hepatobiliar, respiratoria, urinaria, genital, gastroentérica y ósea. La asociación de la variable hacinamiento será evaluada en los pacientes con infección respiratoria (BM, 1993); vasculopatía, neuropatía periférica y autonómica general dentro de infecciones de piel y partes blandas (ADA, 1999); y vejiga neurogénica (neuropatía autonómica) dentro de infecciones del tracto urinario (Pozzilli et al., 1997).

Las variables independientes a considerar son: - Sociodemográficas: grado de instrucción (analfabeto versus primaria, secundaria y superior) (BM, 1993), y hacinamiento no crítico ( $N$ o personas/ No total habitaciones en la vivienda $>2$, excluyendo baño, cocina y pasadizo) (INEI, 1994).

- Clínicas (diagnostico clínico):

a) Desnutrición: según el Índice de Masa Corporal $(\mathrm{IMC})=$ Peso/Talla2 $\left(\mathrm{kg} / \mathrm{m}^{2}\right)$. Debido a la diferencia en la composición corporal según género, desnutrición será definida cuando el IMC sea menor de 20 en varones y menor de 19 en mujeres (ALAD, 1995).

b) Enfermedad vascular periférica: definida según hallazgos físicos de gangrena, úlceras, ausencia o disminución de pulsos periféricos, antecedente de cirugía vascular, claudicación intermitente o pie frío (ADA, 1999).

c) Neuropatía periférica establecida (excluyendo parestesias del diagnóstico clínico por su potencial reversibilidad): definida clínicamente como neuropatía dolorosa, hipo o arreflexia profunda (aquileano, patelar), hipotrofia de músculos interóseos, síndrome del túnel del carpo, pie caído, mononeuropatía craneal, neuropatía toracoabdominal, déficit sensitivo protopático, vibratorio (medido con diapasón) o posicional (ALAD, 1995; Levin \& Pfeifer, 1998).

d) Neuropatía autonómica: definida clínicamente como la presencia de hipotensión ortostática, vejiga neurogénica (disminución de la frecuencia urinaria, infección urinaria a repetición, dificultad para completar la micción, chorro débil, dificultad para iniciar micción, goteo post-miccional e incontinencia por rebosamiento), impotencia sexual, eyaculación retrógrada, enteropatía diarreica y/ o estreñimiento, gastroparesia, incontinencia anorectal, disminución o ausencia de sudoración (Hirsch, 1996; Levin \& Pfeifer, 1998).

- Laboratoriales:

a) Relación albúmina/globulina séricas (A/G): <1, patológica (Rubin, 1995). 
b) Creatinina sérica (retención nitrogenada): los valores de normalidad en el laboratorio central del $\mathrm{HNCH}$ son de $0,7-1,1 \mathrm{mg} / \mathrm{dl}$ en hombres y de 0,6-0,9mg/ dl en mujeres.

Las variables a emplear descriptivamente son: - Duración de diabetes o Tiempo de enfermedad: considerada desde el momento en que el paciente recibe un diagnóstico de diabetes tipo 2 por un profesional médico.

- IMC: en varones y mujeres, obesidad fue definida según IMC $\geq 27$ e IMC $\geq 26$, sobrepeso según IMC > 25 y <27 e IMC > 24 y <26; e IMC normal de $20-25$ y $19-24 \mathrm{~kg} / \mathrm{m}^{2}$, respectivamente (ALAD, 1995).

- Hemoglobina glicosilada: como monitoreo de control metabólico se emplearon los dos primeros años del periodo elegido, valores de HbA1 total (sólo durante los dos a tres meses previos a la hospitalización por ser una base reversible con los cambios de glicemia) y HbAlc, por razones logísticas durante los últimos ocho años del estudio, pudiendo tomarse incluso durante la hospitalización. Según análisis por columna de resinas y recomendaciones de la Asociación Latinoamericana de Diabetes (ALAD), se catalogarán los valores en rango diabético aceptable a HbA1: 8,1-10\% y Alc: 6,6-7,5\% y como mal control a $\mathrm{HbA} 1>10 \%$ y $\mathrm{HbAlc}>7,5 \%$ (ALAD, 1995).

\section{Análisis estadístico}

Se llevó a cabo mediante software estadístico (Epi Info - Dean et al., 1994 - y SPSS - SPSS Incorporation, 1999). Se realizaron pruebas de significancia de Chi cuadrado (en algunos casos con análisis de tendencia lineal en proporciones), con corrección de Fisher para algunos casos. Se realizó un análisis bivariado con postestratificación según dicotomización de edad (60 años de edad por medidas de tendencia central al no encontrarse asociación entre un punto definido de edad e infecciones) y sexo, de aquellas variables previamente identificadas en la literatura y obteniéndose los valores de OR controlados. Las variables de interés significativas obtenidas fueron evaluadas en un análisis multivariado stepwise de tipo forward con el consiguiente ajuste del valor de OR según el modelo de mejor ajuste en infección de piel y partes blandas, incluyendo al modelo edad, sexo, vasculopatía, neuropatía periférica y autonómica general. Se trabajó con un nivel de significancia de un valor de $p \leq 0,05$. Por la limitada frecuencia de la variable vejiga neurogénica, no fue posible realizar el análisis multivariado en infecciones urinarias.

\section{Resultados}

El total de pacientes incluidos en el estudio fue 210. El total de casos resultaron ser infecciones adquiridas en la comunidad sin un antecedente hospitalario en nuestro centro por haberse seleccionado la primera hospitalización y sin reemplazo. Se encontró distribución homogénea entre los casos y controles de las variables sociodemográficas, lo cual favorece la comparabilidad entre ellos respecto a las variables de investigación (Tabla 1). Casi la totalidad de los pacientes incluidos fueron de origen mestizo. La variable grado de instrucción no mostró significancia estadística al análisis de tendencia lineal en proporciones.

Los motivos de hospitalización según grupo caso y grupo control se muestran en la Tabla 2.

Entre los episodios de infección adquirida en la comunidad encontrados en el grupo de casos estuvieron la infección de piel y partes blandas $(43,8 \%)$, urinaria $(40 \%)$, respiratoria $(20 \%)$, gastrointestinal $(6,7 \%)$ y otros (hepatobiliar, meningoencefálica, ósea, ocular y ótica) en un $7,6 \%$.

La duración de la diabetes en el grupo de casos y controles fue de 11,393 $\pm 9,14$ años y 10,664 $\pm 9,34$ años, respectivamente, no hallándose diferencia significativa entre las medias por ANOVA $(p=0,536)$.

No se halló significancia estadística al análisis de tendencia lineal en proporciones de las variables grado de instrucción eíndice de masa corporal. En el análisis de asociación con la prueba de significancia de Chi cuadrado para las variables en investigación se encontró asociación estadísticamente significativa entre vasculopatía, neuropatía periférica y autonómica (vejiga neurogénica) y pacientes con infección (Tabla 3).

Entre las variables de laboratorio, la inversión al búmina/globulina séricas fue observada en el $49,4 \%$ de los casos ( $n=81$ ) y en el $34,4 \%$ de los controles ( $n=87$ ), con un $p=0,05$ según la prueba de Chi cuadrado. La retención nitrogenada fue observada en el $36,5 \%$ de los casos $(n=104)$ y en el $39 \%$ de los controles $(n=105)$, con un $\mathrm{p}=0,71$ según la prueba de Chi cuadrado. Los totales muestrales fueron diferentes por fuente de datos incompleta.

Dentro de una muestra limitada de 40 pacientes, homogéneamente distribuida ( $p=$ 0,238 ), los valores de hemoglobina glicosilada (Al ó Alc) mostraron valor el evado (mal control metabólico) en el 88,9\% $(n=18)$ de los casos y $68,2 \%(n=22)$ de los controles.

Los resultados del análisis bivariado sin ajuste y estratificado según edad (mayor y me- 
Distribución de las variables sociodemográficas según grupo de pacientes.

\begin{tabular}{|c|c|c|c|c|c|c|}
\hline \multirow[t]{2}{*}{ Variables } & \multicolumn{2}{|c|}{$\begin{array}{c}\text { Pacientes com infección } \\
\text { en general }\end{array}$} & \multicolumn{2}{|c|}{ Pacientes sin infección } & \multirow[t]{2}{*}{$\mathrm{n}$} & \multirow[t]{2}{*}{$\mathrm{p}$} \\
\hline & $\mathrm{n}$ & $\%$ & $\mathrm{n}$ & $\%$ & & \\
\hline \multicolumn{7}{|l|}{ Sexo } \\
\hline Masculino & 60 & 57,1 & 55 & 52,4 & 210 & 0,488 \\
\hline Femenino & 45 & 42,9 & 50 & 47,6 & & \\
\hline \multicolumn{7}{|l|}{ Edad (años) } \\
\hline Media \pm desviación estándar & $58 \pm 11$ & & $59 \pm 11,4$ & & 210 & $0,271^{*}$ \\
\hline Mediana & 58 & & 60 & & & \\
\hline \multicolumn{7}{|l|}{ Estado civil } \\
\hline Soltero o otros & 29 & 27,6 & 35 & 33,3 & 210 & 0,368 \\
\hline Casado o conviviente & 76 & 72,4 & 70 & 66,7 & & \\
\hline \multicolumn{7}{|l|}{ Grado de instrucción } \\
\hline Analfabeto & 10 & 9,5 & 4 & 3,8 & 210 & 0,298 \\
\hline Primaria & 53 & 50,5 & 61 & 58,1 & & $0,096 * *$ \\
\hline Secundaria & 32 & 30,5 & 33 & 31,4 & & \\
\hline Superior & 10 & 9,5 & 7 & 6,7 & & \\
\hline \multicolumn{7}{|l|}{ Hacinamiento*** } \\
\hline Sí & 9 & 42,8 & 33 & 33,3 & 120 & 0,406 \\
\hline No & 12 & 57,2 & 66 & 66,7 & & \\
\hline
\end{tabular}

* Valor de p por ANOVA

** Valor de p comparando analfabeto/otros

*** Seleccionada sólo para casos con infección respiratoria

nor de 60 años) y sexo para las variables de investigación se presentan en la Tabla 4.

Los valores de OR bivariado, estratificado por edad y sexo y multivariado con el mejor ajuste en infecciones de piel y partes blandas, se presentan en la Tabla 5.

Los modelos multivariados con interacción entre vasculopatía y neuropatía periférica o neuropatía periférica y autonómica no mostraron un mejor ajuste. Se aisló a vasculopatía periférica y edad $<60$ años como variables de riesgo (valor de p del modelo $<0,01$ ).

\section{Discusión}

Para este estudio, calculamos una muestra representativa de los diabéticos hospitalizados. Nuestros resultados descriptivos sociodemográficos son similares a los descritos en la población diabética del $\mathrm{HNCH}$, según reportes de Villena \& Romero (1991). El HNCH (hospital universitario de cuarto nivel) se encuentra ubicado al norte de la ciudad de Lima (dos millones de personas de población aproximada),
Tabla 2

Motivo de hospitalización según grupos de estudio.

\begin{tabular}{lr}
\hline Admisión en el grupo con infecciones en general $(\mathrm{n}=105)$ & $\%$ \\
\hline Infección actual únicamente & 52,4 \\
Insuficiencia renal y/o cardiaca congestiva & 34,3 \\
Accidente cerebro vascular & 5,7 \\
Convulsión tardía & 3,8 \\
Otros & 3,8 \\
& \\
\hline Admisión en el grupo de diabéticos sin infección (n = 105) & $\%$ \\
\hline Insuficiencia renal y/o cardiaca congestiva & 22,8 \\
Sd. hiperosmolar por falta de tratamiento & 20,0 \\
Desorden cerebro vascular & 17,1 \\
Hipoglicemia & 10,5 \\
Enfermedad coronaria & 9,5 \\
Úlcera gastrointestinal & 4,8 \\
Arritmia cardiaca & 4,8 \\
Otros & 10,4 \\
\hline
\end{tabular}


Tabla 3

Distribución de las variables clínicas según tipo de paciente.

\begin{tabular}{|c|c|c|c|c|c|c|}
\hline \multirow[t]{2}{*}{ Variables } & \multicolumn{2}{|c|}{ Pacientes con infección } & \multicolumn{2}{|c|}{ Pacientes sin infección } & \multirow[t]{2}{*}{$\mathrm{n}$} & \multirow[t]{2}{*}{$\mathrm{p}$} \\
\hline & $n$ & & $\mathrm{n}$ & & & \\
\hline \multicolumn{7}{|c|}{$\begin{array}{l}\text { Índice de masa corporal } \\
\text { (infecciones totales) }\end{array}$} \\
\hline Desnutrición & 4 & 4,5 & 8 & 8,4 & 184 & 0,088 \\
\hline Normal & 40 & 45,0 & 26 & 27,4 & & $0,435 *$ \\
\hline Sobrepeso & 11 & 12,3 & 15 & 15,8 & & \\
\hline O beso & 34 & 38,2 & 46 & 48,4 & & \\
\hline \multicolumn{7}{|c|}{$\begin{array}{l}\text { Vasculopatía periférica (infección } \\
\text { de partes blandas y óseas) }\end{array}$} \\
\hline Sí & 37 & 80,4 & 50 & 47,6 & 151 & 0,0003 \\
\hline No & 9 & 19,6 & 55 & 52,4 & & \\
\hline \multicolumn{7}{|c|}{$\begin{array}{l}\text { Neuropatía periférica (infección } \\
\text { de partes blandas y óseas) }\end{array}$} \\
\hline Sí & 40 & 88,8 & 76 & 72,4 & 150 & 0,045 \\
\hline No & 5 & 11,2 & 29 & 27,6 & & \\
\hline \multicolumn{7}{|c|}{$\begin{array}{l}\text { Neuropatía autonómica } \\
\text { (infección del tracto urinario) }\end{array}$} \\
\hline Sí & 14 & 33,3 & 11 & 10,5 & 147 & 0,002 \\
\hline No & 28 & 66,7 & 94 & 89,5 & & \\
\hline
\end{tabular}

* Valor de p comparando desnutrición/otros

** Total de casos y controles diferentes por fuente de datos incompleta y selección según tipo de infección

Tabla 4

Valores de odds ratio $(O R)$ bivariado y estratificado por edad y sexo.

\begin{tabular}{lcccc}
\hline Variables & OR & IC $95 \%$ & OR estratificado & IC $95 \%$ \\
\hline Infecciones en general & & & & \\
$\quad$ Grado de instrucción & & & & \\
$\quad$ Analfabeto/otros & 2,66 & $0,73-11,95$ & 2,35 & $0,56-9,45$ \\
$\quad$ Desnutrición & 0,51 & $0,11-2,00$ & 0,40 & $0,09-1,68$ \\
$\quad$ Inversión albúmino/globulina & 1,85 & $0,95-3,62$ & 1,87 & $0,95-3,65$ \\
$\quad$ Creatina sérica & 0,90 & $0,49-1,63$ & 0,93 & $0,51-1,71$ \\
Infecciones respiratorias & & & & \\
$\quad$ Hacinamiento & 1,50 & $0,52-4,32$ & 1,14 & $0,23-4,82$ \\
Infecciones urinarias & & & & \\
$\quad$ Neuropatía autonómica (vejiga neurogénica) & 4,27 & $1,58-11,58$ & 4,07 & $1,45-10,98$ \\
\hline
\end{tabular}


Capital del Perú. Según la Dirección de Salud Lima Norte, la población mayor de 30 años de edad asignada al $\mathrm{HNCH}$ dentro de la región $\mathrm{Li}$ ma Norte es de 33.545 pacientes, la cual representa el 2,4\% del total de los pacientes de la región. El número total de camas disponibles en el $\mathrm{HNCH}$ es de 376, lo cual representa el 32\% del total disponible en la región. El $27 \%$ de las camas están asignadas al servicio de medicina interna y tropical. EI HNCH así como la red de hospitales nacionales, cuenta con el programa de clínica de diabetes. El Ministerio de Salud tiene asignado al $\mathrm{HNCH}$ a 558 diabéticos tipo 1 y 2 para su atención en la región norte.

Por razones de costos y tipos de controles (hospitalarios) empleados no evaluamos muchos otros factores de riesgo potencial de infecciones como grado de inactividad física, hiperglicemia, duración de la enfermedad, saneamiento ambiental, nivel socioeconómico, entre otros; sin embargo, hemos considerado aquellos factores frecuentes, asociados al desarrollo de infecciones y factibles de rápida detección en diabéticos que acuden a la consulta médica ambulatoria u hospitalaria.

Se describe la mayor incidencia de enfermedades en general (incluyendo diabetes) entre estratos de nivel socioeconómico (NSE) y de instrucción inferior (BM, 1993). El nivel de instrucción de la población diabética atendida en el HNCH es mayormente primario (Villena \& Romero, 1991), lo cual explicaría la comparable distribución de frecuencias del nivel educativo analfabeto y primario entre los grupos estudiados. Son estos grupos los menos adherentes al tratamiento, con el consiguiente descontrol metabólico (Cabrera et al., 1996). Sin embargo, se observó la existencia de un grupo de pacientes con educación superior entre los diabéticos infectados, lo cual nos sugeriría el utilizar iguales recursos de educación preventiva independientemente del nivel de educación. En nuestro análisis observamos sólo una aparente asociación positiva entre analfabetismo e infecciones, la cual se podría confirmar en un población con mayor representación de los diferentes niveles de instrucción.

El antecedente sociodemográfico de hacinamiento, evaluado desde un nivel no crítico, se encontró en relativa frecuencia en ambos grupos de comparación concordante con el NSE bajo a medio de la población estudiada (Villena \& Romero, 1991), subestimando, sin embargo, su efecto evaluado sobre el grupo con infecciones respiratorias (BM, 1993). Su discusión es de importancia por encontrarse asociado a infecciones como Tuberculosis Pulmonar, la cual ocupa entre el segundo y el ter-
Tabla 5

Valores de odds ratio (OR) bivariado, estratificado por edad y sexo, y multivariado en infección de piel y partes blandas.

\begin{tabular}{lccc}
\hline $\begin{array}{l}\text { Infección piel y } \\
\text { partes blandas }\end{array}$ & $\begin{array}{c}\text { OR crudo } \\
\text { (IC 95 \%) }\end{array}$ & $\begin{array}{c}\text { OR estratificado } \\
\text { (IC 95\%) }\end{array}$ & $\begin{array}{c}\text { OR multivariado } \\
(\text { IC 95\%) }\end{array}$ \\
\hline Vasculopatía periférica & 4,52 & 5,74 & 6,79 \\
Neuropatía periférica & 3,05 & $(2,20-15,20)$ & $(2,75-16,79)$ \\
& $(1,05-10,81)$ & $(1,14-12,61)$ & $10,45-4,83)$ \\
Neuropatía autonómica & 1,83 & 1,90 & 1,55 \\
& $(0,85-3,94)$ & $(0,85-4,25)$ & $(0,68-3,55)$ \\
\hline
\end{tabular}

$* \mathrm{R}^{2} \mathrm{~N}$ agelkerke $=0,242$

cer lugar en frecuencia entre las infecciones presentes en diabéticos tipo 1 y 2 de hospitales de Lima (Villena et al., 1996).

Entre las características clínicas estudiadas, hemos hallado una menor proporción de pacientes con sobrepeso y obesidad y mayor proporción con desnutrición que la esperada según se describe para diabetes tipo 2 (Foster, 1998), explicada por el aumento de pérdida de energía debido a los procesos intercurrentes y el mal control metabólico (inferido a partir de un pequeño número de observaciones para dicho factor debido a bajos recursos económicos de nuestra población) encontrados similarmente entre los grupos de estudio, lo cual a su vez explicaría la subvaloración de la predisposición a infecciones por inmunosupresión secundaria a desnutrición (Tramont \& Hoover, 1995).

La Enfermedad Vascular Periférica (EVP) predispone a infecciones de partes blandas en diabéticos en general, por ocasionar isquemia y ulceración de partes blandas así como alterar la función oxígeno dependiente de los polimorfonucleares (Currie et al., 1998). La EVP se reporta como factor asociado a infecciones en diabéticos (Concha, 1995; Marulanda \& De Martinez, 1989). Nuestros resultados aislaron independientemente a la EVP como contribuyente a infecciones de piel y partes blandas, mostrando además el efecto confusor de factores como edad y sexo al análisis estratificado, por la descrita asociación entre el desarrollo de enfermedad cardiovascular y sexo femenino o mayor edad (Nathan, 1993). Sin embargo, en el modelo multivariado con mejor ajuste y significativo, se pudo observar el efecto protector de edades mayores a 60 años frente a las infeccio- 
nes de piel y partes blandas, lo cual se explicaría por el hecho de que la mayoría (73\%) de cirugías de amputación de miembros se realizaron en nuestra serie de casos antes de los 60 años de edad por procesos sépticos de difícil control a partir de infecciones de partes blandas, cuadro que también se reporta en series extranjeras (Currie et al., 1998; Humphrey et al., 1996).

Neuropatía autonómica, en general, fue incluída al modelo multivariado por la probable presencia de disfunción sudomotora y potencial alteración de la piel como barrera de defensa, así como la factible diseminación hematógena de enterobacterias a partir de focos urinarios e intestinales con sobrecrecimiento bacteriano asociada a desordenes autonómicos secundarios a diabetes (Ryan \& Sleisenger, 1993).

Secundariamente al desarrollo de complicaciones neuropáticas, las infecciones de piel y partes blandas y aparato urinario son las más frecuentes en diabéticos a nivel global (Contreras, 1993; Villena et al., 1996), lo cual hemos observado en nuestros resultados. Los daños neuropático periférico y autonómico pueden indirectamente provocar infecciones de partes blandas por el desarrollo secundario de úlceras cutáneas por pérdida de sensibilidad y deformación del pie y disfunción sudo motora, respectivamente (Hirsch, 1996; Pozzilli et al., 1997). En congruencia con una publicación extranjera (Marulanda \& De Martinez, 1989), se halló como factor asociado positivamente a infecciones de piel y partes blandas al daño neuropático periférico en el análisis bivariado y estratificado por edad y sexo. Los factores edad y sexo mostraron ser de confusión durante el análisis estratificado, explicado por la asociación del sexo masculino y mayor edad al desarrollo de neuropatía periférica (Hirsch, 1996). En el análisis multivariado, aunque con menor ajuste, se pudo observar interacción entre neuropatía y vasculopatía periféricas, como sería de esperar en el proceso fisiopatológico del pie diabético (Eliopoulos, 1995); sin embargo, el aislamiento de neuropatía periférica independientemente no fue observado, creemos, por la falta de ajuste adicionalmente de factores de confusión como desnutrición y mal control metabólico que puedan mediar su efecto y sesgos propios del estudio (Schlesselman, 1982).

En la selección de casos y controles con vejiga neurogénica, encontramos al análisis estratificado según edad y sexo asociación positiva altamente significativa ( $p=0,004)$ a infecciones urinarias, confirmando dicha asociación reportada internacionalmente (Eliopulos, 1995). En el futuro, sugerimos en un análisis multivariado el control de otros potenciales factores asociados al desarrollo de neuropatía autonómica o correlacionados a una mayor incidencia de infecciones urinarias en diabéticos (Eliopulos, 1995; Hirsch, 1996).

La enfermedad avanzada, traducida en las frecuentes complicaciones observadas en nuestra población hospitalaria, explicaría la falta de asociación obtenida entre inversión de la relación albúmina/globulina séricas (asociada a desnutrición) y retención nitrogenada, ambas asociadas al daño renal por diabetes, e infecciones en general, como describe Rubin (1995), por representar estados de inmunosupresión secundaria.

En base a nuestra experiencia y a revisión bibliográfica, planteamos la necesidad de evaluar a futuro el grado de asociación del daño sudo motor a infección de partes blandas, el efecto dosis respuesta de la elevación del nivel de creatinina y descripción ordinal del hacinamiento y rol predictivo de factores relacionados al daño macroangiopático como duración de diabetes, hiperglicemia crónica, dislipidemia, hipertensión y microalbuminuria, en el desarrollo de infecciones de partes blandas y urinarias.

Dentro de las limitaciones propias del diseño retrospectivo de nuestro estudio y con intención de que se logre a futuro un mejor diseño, sugerimos elegir un mayor poder estadístico del estudio, que permita ajustar las variaciones de los OR para aquellos factores que no fueron significativos en nuestro análisis, confirmar laboratorial mente los diagnósticos clínicos, continuar el estudio de nuestras hipótesis en un diseño prospectivo y seleccionar controles poblacionales validados.

En conclusión, se debe señalar a partir de nuestro estudio que la vasculopatía periférica y la vejiga neurogénica contribuyen al desarrollo de infecciones de piel y partes blandas y urinarias, respectivamente, adquiridas en la comunidad, en diabéticos tipo 2, hospitalizados. 


\section{Referencias}

ADA (American Diabetes Association), 1999. Standards of medical care for patients with diabetes mellitus. Diabetes Care, 22(Sup. 1):S32-S41.

ALAD (Asociación Latinoamericana de Diabetes), 1995. Consenso sobre Prevención, Control y Tratamiento de la Diabetes Mellitus no Insulinodependiente. Buenos Aires: Ediciones Mayo.

BALKAU, B.; ESCHWÉGE, E.; PAPOZ, L.; RICHARD, J.-L.; CLAUDE, J.-R.; WARNET, J.-M. \& DUCIMETIÉRE, P., 1993. Risk factors for early death in non-insulin dependent diabetes and men with known glucose tolerance status. BMJ, 307:295-299.

BM (Banco Mundial), 1993. Informe sobreed Desarrollo Mundial. Invertir en Salud. Indicadores del Desarrollo Mundial. Washington, DC: BM .

CABRERA, C.; MARTINEZ, A.; VEGA, M.; GONZALES, G. \& MUÑOZ, A., 1996. Prácticas nutricias en pacientes diabéticos tipo II en el primer nivel de atención. Instituto Mexicano del Seguro Social (IMSS), Jalisco, M éxico. Cadernos deSaúde Pública, 12:525-530.

CONCHA, P., 1995. Infecciones en Pacientes con Diabetes Mellitus Hospitalizados en el Hospital Nacional San Agustín. Tesis de Bachiller en Medicina, Arequipa: Facultad de Medicina, Universidad Nacional San Agustín.

CONTRERAS, S., 1993. Causas de muerte en pacientes diabéticos. Revista de la Asociación LatinoAmericana de Diabetes (ALAD), 1:18-21.

CURRIE, C. J.; MORGAN, C. L. \& PETERS, J. R., 1998. The epidemiology and cost of inpatient care for peripheral vascular disease, infection neuropathy and ulceration in diabetes. Diabetes Care, 21:42-48.

DE AGUILAR, L. G.; CARNEIRO, J. R.; GINZBARG, D.; CUNHA, E. F. \& GOMES, M. B., 1997. Infecção diagnosticada em diabéticos durante internação hospitalar. Revista da Associação Médica Brasileira, 43:314-318.

ELIOPOULOS, G., 1995. Infections in diabetes mellitus. Infectious Disease Clinics of North America, 9:1-221.

FILE Jr., T. M. \& TAN, J. S., 1997. Infectious complications in diabetic patients. Current Therapy in Endocrinology Metabolism, 6:491-495.

FORE, W., 1995. Diabetes insulinoindependiente. Prevención de complicaciones. Medical Clinics of North America, 2:297-307.

FOSTER, D. W., 1998. Diabetes mellitus. In: Harrison's Principles of Internal Medicine (A. Fauci \& D. Longo, ed.), pp. 2060-2081, New York: McGraw-Hill Press.

GARNER, J. S.; JARVIS, W. R. \& EMORY, T. G., 1988. CDC definitions for nosocomial infections. American Journal of Infection Control, 16:128-140.

HIRSCH, I. B., 1996. Surveillance for complications of diabetes. Postgraduate Medicine, 99:147-161.

HUMPHREY, A. R. G.; DOWSE, G. K.; THOMA, K. \& ZIMMET, P. Z., 1996. Diabetes and nontraumatic lower extremity amputations. Incidence, risk factors, and prevention-a 12-year follow-up study in Nauru. Diabetes Care, 19:710-714.

INEI (Instituto Nacional de Estadística e Informática), 1994. Perú: Mapa de Necesidades Bási cas Insatisfechas de los Hogares a Nivel Distrital. Aspec- tos Conceptuales y Metodológi cos dela Medición de la Pobreza. Lima: Dirección Técnica de Demografía y Estudios Sociales.

LASKY, T. \& STOLLEY, P., 1996. Selección de casos y controles. Boletín de la Oficina Sanitaria Panamericana, 120:441-453.

LEVIN, M. E. \& PFEIFER, M. A., 1998. Uncomplicated Guide to Diabetes Complications. Alexandria: American Diabetes Association.

MALERBI, D. A. \& FRANCO, L. J., 1992. Multicenter study of the prevalence of diabetes mellitus and impaired glucose tolerance in urban Brazilian population aged 30-69 yr. The Brazilian Cooperative Group on the Study of Diabetes. Diabetes Care, 15:1509-1516.

MARULANDA, M. \& DE MARTINEZ, M., 1989. Infección en el Paciente Diabético. Tesis de Especialista, Carabobo: Facultad de Medicina, Universidad de Carabobo.

NATHAN, D. M., 1993. Long-term complications of diabetes mellitus. New England Journal of Medicine, 328:1676-1683.

PFIZER, 1982. Manual de Diabetes Mellitus. San José: Pfizer.

POZZILLI, P.; SIGNORE, A. \& LESLIE, R., 1997. Infections, immunity and diabetes. In: International Textbook of Diabetes Mellitus (K. Alberti, P. Zimmet, R. De Fronzo \& H. Keen, ed.), pp. 1231-1241, New York: John Wiley \& Sons.

RUBIN, R. H., 1995. Infection in the immnunosupressed host. In: Scientific American MedicineBulletin (M. Rubenstein \& R. Federman, ed.), pp. 123, New York: Scientific American.

RYAN, J. \& SLEISENGER, M., 1993. Effects of systemic and extra intestinal disease on the gut. In: Gastrointestinal Disease (M. Sleisenger \&J. Fordtran, ed.), pp. 193-239, Philadelphia: W. B. Saunders Company.

SCHLESSELMAN, J. J., 1982. Case-Control Studies. New York: Oxford University Press.

SECLEN, S.; LEEY, J.; VILLENA, A.; HERRERA, B.; MENACHO, J.; CARRASCO, A. \&VARGAS, R., 1999. Prevalencia de diabetes mellitus, hipertensión arterial, hipercolesterolemia y obesidad como factores de riesgo coronario y cerebrovascular en población adulta de la costa sierra y selva del Perú. Acta Médica Peruana, 17:8-12.

TRAMONT, C. E. \& HOOVER, L. D., 1995. General or non specific host defense mechanisms. In: Principles and Practice on Infectious Diseases (G. Mandell, J. Benett \& R. Dolin, ed.), pp. 30-35, New York: Churchill Livingstone.

VILLENA, J.; BURGA, J.; CORIGLIANO, S. \& VALDIVIA, J., 1996. Morbimortalidad por diabetes mellitus no insulino dependiente (DMNID) en el Hospital Nacional Cayetano Heredia, 1985-1995. Revista Médica del Perú, 68:64.

VILLENA, J. \& ROMERO, S., 1991. Características socioeconómicas y culturales de los pacientes diabéticos no insulinodependientes del Hospital Nacional Cayetano Heredia. Diagnóstico, 28:93-97. 